\title{
Política nacional de igualdad de género y el currículo nacional de la educación básica regular en el norte del Perú
}

\author{
Elizabet Segura Vásquez \\ elizabetsegura.152@gmail.com \\ https://orcid.org/0000-0001-8459-3170 \\ Universidad César Vallejo, Lima, Perú \\ Graus Cortez Lupe Esther \\ gracortez@ucvvirtual.edu.pe \\ https://orcid.org/0000-0002-1511-5244 \\ Universidad César Vallejo, Lima, Perú \\ Lindon Vela Meléndez \\ lvela@unprg.edu.pe \\ https://orcid.org/0000-0002-9644-715 \\ Universidad Nacional Pedro Ruiz Gallo, Lambayeque, Perú \\ León Velarde César Gerardo \\ cesoar@hotmail.com \\ https://orcid.org/0000-0002-8273-1995 \\ Universidad César Vallejo, Lima, Perú \\ Merino Flores Irene \\ imerinof@ucvvirtual.edu.pe \\ https://orcid.org/0000-0003-3026-5766 \\ Universidad César Vallejo, Lima, Perú \\ Panche Rodríguez Odoña Beatriz \\ Opancher@ucv.edu.pe \\ https://orcid.org/0000-0002-1629-1776 \\ Universidad César Vallejo, Lima, Perú \\ Samamé Cornejo César Artidoro \\ samame.uss@gmail.com

$\begin{array}{r}\text { Samamé Cornejo César Artidoro } \\ \text { samame.uss@ @mail.com }\end{array}$
$\frac{\text { https://orcid.org/0000-0002-7057-0012 }}{\text { Universidad César Vallejo, Perú }}$

\section{RESUMEN}

En un país que se encuentra en plena modernización de la gestión pública, donde las Políticas de Estado y de Gobierno son el primer pilar central en la gestión del cambio, la educación juega una labor determinante en consentir el paso de un enfoque marginal hacia una intervención diligente; en ese sentido, el presente estudio, tiene por objetivo determinar el nivel de influencia de la política nacional de igualdad de género en el 
currículo de la educación básica regular en la Región Lambayeque, para lo cual se utilizó la técnica de la encuesta y la entrevista, para luego, con los resultados obtenidos realizar el análisis en el programa SPSS 25, que permitieron obtener la validación de la hipótesis que establece que la política nacional de igualdad de género tiene influencia positiva en el currículo nacional de la educación básica regular en la Región Lambayeque. Por lo que se concluyó que, de acuerdo a los resultados obtenidos, la existencia de una correlación alta, y positiva, entre las variables de estudio, debido al coeficiente de correlación de Spearman.

Palabras clave: política pública; enfoque de género; educación básica regular; desigualdad; brechas de género. 


\title{
National policy on gender equality and the national curriculum for regular basic education in northern Peru
}

\begin{abstract}
In a country that is in the midst of modernizing public management, where State and Government Policies are the first central pillar in the management of change, education plays a decisive role in allowing the transition from a marginal approach to a diligent intervention; In this sense, the present study aims to determine the level of influence of the national policy of gender equality in the curriculum of regular basic education in the Lambayeque Region, for which the survey and interview technique was used, and then, with the results obtained, the analysis was carried out in the SPSS 25 program, which allowed obtaining the validation of the hypothesis that establishes that the national policy of gender equality has a positive influence on the national curriculum of regular basic education in the Lambayeque Region. Therefore, it was concluded that, according to the results obtained, there is a high and positive correlation between the study variables, due to Spearman's correlation coefficient.
\end{abstract}

Keywords: public policy; gender focus; regular basic education; inequality; gender gaps.

Artículo recibido: 10. junio. 2021 Aceptado para publicación: 20. julio. 2021 Correspondencia: elizabetsegura.152@gmail.com Conflictos de Interés: Ninguna que declarar 


\section{INTRODUCCIÓN}

La desigualdad de género ha sido por cientos de años una disfunción social que genera estancamiento en el progreso de nuestra sociedad, es por ello que se idearon y normalizaron derechos y deberes para que todos y todas viviéramos en un mundo equilibrado, desterrando para ello, patriarcados y estereotipos implantados de toda la vida. Es pertinente señalar, que la importancia de contar con políticas públicas que progresivamente se han ido enfocando en el género, va más allá de realizar una diferenciación entre varones y mujeres, básicamente se centra en la implicancia de esa diferencia, en las desigualdades de género de las personas, en sus necesidades por razón de su género, en las demandas y experiencias que estas implican; en razón de esto, elaborar una estrategia pública con enfoque de género, que tenga como finalidad fundamental apuntar la igualdad de posibilidades para varones y mujeres así como desterrar los estereotipos y la discriminación que se ha venido heredando desde los orígenes de las sociedades.

Es extensamente conocido que, en un proceso de reforma, la educación juega una labor definitiva en consentir el paso de un enfoque marginal hacia una en la que se compruebe una intervención diligente; no obstante, en la mayoría de las sociedades se encuentran obstáculos, absurdos sociales y culturales que reducen el ingreso de la mujer a los servicios educativos.

En ese sentido, es importante la creación de un ambiente educacional y social en el que se busque la equidad entre las mujeres y los varones, las niñas y los niños; en el que se los desafíe a impulsar su capacidad al máximo, honrando su libertad de pensamiento, razón, precepto y dogmas, y en el que los recursos educacionales fomenten modelos no estereotipadas de las damas y de los varones, lo que coadyuvaría de orden acertado en aniquilar las razones de la discriminación frente a las mujeres y los desequilibrios entre los mismos, para hacer una lucha frontal a la desigualdad de acceso a la preparación y a los beneficios educacionales, que son incompletos, por lo que, nuestros gobernantes y otros operadores sociales deberían fomentar una política eficaz y notable de unificación en la contingencia de género, en todas las políticas, estrategias, planes y programas, siendo que el compromiso de un futuro mejor, ha requerido y necesita, aún, del esfuerzo de todos y todas. 
De esta manera, la investigación, aborda la implementación de una Política Nacional de Igualdad de Género - PNIG siglas en adelante, y cómo ésta influye en el Currículo Nacional de la Educación Básica Regular - CNEBR siglas en adelante, en el Perú, partiendo de que la diferenciación por discursos de género configura un embarazo para el derecho a la educación y el progreso íntegro de las sujetos, a manera de ejemplificar, en instituciones educativas aún se sigue impartiendo enseñanzas como: que existen carreras y oficios que sólo son para varones y profesiones que sólo la pueden desempeñar las mujeres por su condición como tales, que en el grado inicial, las maestras adviertan a niños a juguetear con carritos y a las niñas con una cocinita o muñecas, así mismo, se realce a un niño como líder y a una niña con las mismas cualidades como mandamás, que si un niño lloriquea es "maricón", que si una niña juega futbol es "machona", entre otras aseveraciones sin fundamento, que incentivan el desequilibrio y normalizan conductas machistas.

En la Región Lambayeque, Perú, con sus tres distritos Chiclayo, Ferreñafe y Lambayeque, cuenta, según el Portal Web del Gobierno Regional de Lambayeque, (s.f.), con setecientos setenta y cuatro (774) instituciones educativas públicas y privadas, donde el 60\% de éstas se encuentran en el sector rural, por lo que hay la necesidad que la PNIG, quede clara y se implemente de manera eficaz en el CNEBR, en aras del bienestar estudiantil de la región.

De esta manera, después de abordar la realidad problemática y la necesidad de la investigación, es indispensable formular el problema del estudio de investigación, en la siguiente interrogante: ¿Cuál es el nivel de influencia de la política nacional de igualdad de género en el currículo nacional de la educación básica regular en Lambayeque?, para la resolución de la misma, es importante justificar la presente investigación, amparándonos en la justificación metodológica y práctica que en los párrafos siguientes se explican.

Se implementará una justificación metodológica que coadyuve a la recolección de datos en un enfoque cualitativo, tomando en cuenta que no se ha identificado en Perú y el mundo, una técnica pura y no se halla un texto o sumario para forjar el análisis de la implementación de la PNIG y cómo ésta influye en el CNEBR de manera que, a través de esta destreza, se innovará una táctica metodológica de análisis para estudiar apropiadamente a la muestra. 
De igual manera, es necesaria una justificación práctica, para resolver la posible existencia de una distorsión del enfoque de género en el CNEBR en la Región Lambayeque, tal como se observa en la situación problemática, al no tener un panorama claro sobre la implicancia de la PNIG, en el CNEBR, en ese sentido, el presente estudio de investigación logrará analizar este problema latente y trascendente en la Región Lambayeque.

Lo antes desarrollado, va a permitir en la investigación, obtener como objetivo general, determinar el nivel de influencia de la política nacional de igualdad de género en el currículo de la educación básica regular en la Región Lambayeque.

De igual manera, las variables que se identifican, permitirá como objetivos específicos, i) distinguir las características de la política pública de igualdad de género en la Región Lambayeque, es también importante, ii) analizar la implementación de la política nacional de igualdad de género, de igual manera, es muy necesario iii) seleccionar las teorías que influyen en la implementación de la política nacional de igualdad de género y el sistema educativo en la Región Lambayeque y por último con el cuarto objetivo específico, se logrará iv) examinar los factores influyentes en la relación entre la política nacional de igualdad de género y el currículo nacional educación básica regular en la Región Lambayeque.

Ello conlleva a plantear la siguiente hipótesis, la política nacional de igualdad de género tiene nivel alto de influencia positiva en el currículo nacional de la educación básica regular en la Región Lambayeque.

\section{Análisis teórico relevante para la investigación.}

Es necesario considerar el sustento teórico en el abordaje del problema de investigación, es así como se considera la Teoría Sociocultural, sustentada por Vygotsky, (1996) quien afirma que: El proceso de los seres humanos, está profundamente unido a su interrelación con el entorno social, histórico y cultural, siendo que a través de ello se cumplen funciones psicológicas de modo óptimo, idóneas del ser humano, como son la memoria, la observación voluntaria, el raciocinio y la resolución de taras, de igual manera, sostiene esta teoría que el desarrollo y desenvolvimiento ontogenético de la psiquis del varón está resuelto por las diversas fases de adquisición de las formas históricas y sociales de la cultura; así mismo, esta teoría opta por afirmar que la elocución obedece a una utilidad conciliadora en este proceso en las fases del desarrollo, en pocas palabras, se puede llegar 
al análisis, que todas las funciones superiores del pensamiento son definitivamente el desenlace de las relaciones interpersonales y sociales, la persona humana se confecciona a sí mismo, a través de la interacción social, citado por (Gutiérrez Rocha et al., 2017)

En ese contexto, para el florecimiento de esta concepción de grado superior es sumamente indispensable que la metodología educativa sea apegadas a cada realidad para otorgar principalmente a la resolución de los asuntos del escenario, las obligaciones y perspectivas de la misma, en ese sentido es inevitable una práctica pedagógica con una representación política y social organizada que conlleve el cambio que se requiere en la sociedad, en razón de ello, el maestro deberá adecuar su práctica educativa precisando la realidad, tomando en cuenta también la clase de alumno, sus aprendizajes previos, grado de madurez, para preparar su lección.

A su vez, en un estudio hecho desde la Universidad Tecnológica de Dublín en Irlanda, sobre las Teorías del Aprendizaje, podemos contextualizar a nuestra realidad y estudio a la Teoría del Aprendizaje Social fundamentada por Merriam y Caffarella en 1999, que si bien es cierto, clasifican esta como una teoría que va de la mano de las teorías del constructivismo, humanismo, conductismo y cognitivismo, no obstante, otros escritores no lo hacen, tanto es así que Tennant (1997) señala que la teoría del aprendizaje social abarca una amplia gama de teorías y enfoques, este autor, llama a esta teoría la perspectiva del "entorno social", para lo cual han surgido dos perspectivas opuestas centradas en la participación activa o pasiva del aprendiz en el proceso de aprendizaje (Ashworth et al., 2004):

Primero, la persona puede ser vista como un receptor pasivo de comportamiento, roles, actitudes y valores que son formados $\mathrm{y}$ mantenidos por el entorno social. La respuesta al estímulo de Skinner, donde la psicología es el más influyente de estos enfoques conductistas (Tennant, 1997). Su impacto en la educación de tercer nivel, es evidente en el establecimiento de objetivos de comportamiento y la provisión de retroalimentación regular y refuerzo a los estudiantes (Stapleton, 2001). El segundo enfoque proporciona un papel activo para la persona. Este enfoque es esencialmente humanista y observa el proceso como dialéctico mediante el cual la persona, la sociedad y el entorno está activo en 
el proceso, en ese sentido, este enfoque puede ser demostrado por los escritos de Freire que observaron los procesos sociales a medida que configuran la identidad individual. Él destacó la necesidad de que los estudiantes adultos resistan las formas de enculturación que son alienantes y opresivos Este enfoque impacta en aprendizaje de tercer nivel en las esferas del aprendizaje permanente, el aprendizaje informal, el aprendizaje experimental y aprendizaje colaborativo (p. 7).

Por otra parte, es vital para la investigación, desarrollar el enfoque de derechos humanos en las diversas políticas de género, para ello, (Meza Pavletich, 2016, p. 29) explica muy bien este enfoque:

Queda claro que los derechos los seres humanos tienen su origen en la exploración del valor de la dignidad y la libertad de la persona autónomamente de la correspondencia que las naciones pudieron haber realizado de los mismos. Por lo que, el análisis y la proyección de las políticas públicas sobre todo en igualdad de la mujer no se ve importante y resaltante, sino hasta que recién cuando el Estado Peruano incluye esta temática en su almanaque público; no cabe duda, es muy factible que la labor antepuesta en esas políticas fuesen los razonamientos indispensables para obtener la mencionada inclusión. Esto quiere decir que al respetar los derechos humanos en el ámbito supranacional requiere de un esfuerzo por trabajar en sus principios, más aún si una sociedad no los respeta íntegramente.

Tal como se explica, defender el enfoque de derechos humanos en las políticas públicas, es un proceso positivo que reconoce empoderar bilateralmente ambas herramientas; además de perfeccionar carencias programáticas y fijar vigencia de los principios del desarrollo originando a través la regla internacional una manera aún más sostenible e inclusiva para el desarrollo.

Es necesario también, para el abordaje de la PNIG, en relación con la CNEBR, el enfoque feminista, para ello, los fundamentos que diseña Patricia Castañeda (2015) en relación con el desarrollo de la educación e investigación con una visión feminista. Por lo que, desde este enfoque, se pretende señalar que la investigación es perpetuar en una colección de procedimientos con orientación a otorgar contestación a las interrogantes superpuestas, con el objetivo de comprender o describir un hecho, sea cual sea la naturaleza de este. En 
ese sentido, recalca que, la investigación feminista, apareció ante la urgencia de hallar la condición de las mujeres, valga decir también su exclusión dentro del dominio científico (Huerta Rojas, 2017, p. 70).

Lo antes desarrollado, lleva a conocimiento de la finalidad esencial de la investigación feminista que es producir discernimientos que logren dar cuenta, entre otras temáticas, del embrollo de las circunstancias de género que implican a mujeres y varones.

\section{ESTRATEGIAS METODOLÓGICAS O MATERIALES Y MÉTODOS}

La investigación tiene un enfoque mixto con énfasis en lo cuantitativo.

En la ruta cuantitativa se tiene un diseño no experimental de corte transversal y en la ruta cualitativa se ha optado por un diseño fenomenológico porque estuvo fundamentado en la observación de disertaciones y contenidos, en especial donde se pretende explorar, puntualizar y comprender los fenómenos desde una perspectiva individual y se aprecia la experiencia de las personas (Fuster Guillen, 2019), en el caso del estudio esta información fue obtenida mediante entrevistas hechas a expertos en educación básica regular de la Región Lambayeque.

La población está conformada por el Consejo Regional por la Igualdad de Género de la Región Lambayeque - COREPIG, los directores de las Unidades de Gestión Educativa Local - Lambayeque, los directores de Gestión Pedagógica en el departamento de Lambayeque, Especialistas de Educación y Jefe de la Unidad Territorial en Lambayeque del Programa Nacional AURORA - MIMP.

A nivel mundial, este año es crucial para la investigación, en razón del distanciamiento social, por la pandemia mundial que tiene como raíz a un virus llamado COVID-19, o Nuevo coronavirus de Wuhan (Cuero, 2020, p. 1), que ha obligado a nuestra sociedad en su conjunto, aislarnos en nuestra casa, lo que hace imposible aplicar los instrumentos de recolección de datos al 100\% de los integrantes de la muestra, por ello, el muestreo utilizado es no probabilístico, por conveniencia, es decir, se aplicarán los instrumentos de recolección de datos solo a casos disponibles, a los cuales se tiene acceso vía redes sociales y plataformas virtuales, en ese sentido, los criterios de inclusión serían: personas con acceso a internet, en el caso de los funcionarios y servidores que tengan un mínimo de experiencia de un año en el ejercicio de sus funciones, con excepción de aquellos que por la naturaleza de su designación llevan menos de un año; así mismo, los criterios de 
exclusión abarcaría a: servidores o funcionarios que no tengan el mínimo requerido de experiencia en el ejercicio de sus funciones.

\section{RESULTADOS Y DISCUSIÓN}

Con relación a las características de la política pública de igualdad de género en la Región Lambayeque, de acuerdo a los resultados obtenidos en la aplicación de la entrevista, se identificaron las siguientes características: La política pública de igualdad de género es transversal, articuladora, orientadora de buenas prácticas, e instructiva. Con relación a la transversalidad de la política, (Soriano, 2020), establece que se debe a que la Política Nacional de Igualdad de género es transversal, definitivamente se incluye el enfoque de género dentro del plan operativo y directivas regionales. Con relación a la característica articuladora, (Nuñez, 2020), establece que es parte de su naturaleza, esto se debe a que, desde el gobierno regional se ha implementado un plan de igualdad de género también tenemos un mecanismo de articulación por ejemplo con el consejo de igualdad y con la comisión técnica de igualdad también de género y con ello se está trabajando y en el marco de la implementación de las políticas es el Estado, que obliga que las instancias se comprometan en sus tres niveles, en la toma de decisiones para alcanzar ese libre ejercicio de derechos y que ninguna persona sea discriminada. Con respecto a su orientación hacia las buenas prácticas, (Jiménez, 2020), opina que, todas maneras, esta política es orientadora de buenas prácticas, por lo que a través de actividades de sensibilización como pasacalles, concursos y otros, se logra llegar al receptor con mensajes y representación gráfica sobre la igualdad que debe haber entre varones y mujeres, desterrando estereotipos o constructos sociales. Con relación a su característica instructiva, a política (Sánchez, 2020), establece que, la PNIG, instruye bastante respecto a la relación que se debe tener en los tres niveles de gobierno, en este caso, entre el Gobierno Regional y la UGEL Lambayeque en específico, se comparte una visión estratégica, objetivos y prioridades políticas, así como conocimiento profundo del territorio, de nuestra diversidad geográfica y cultural, para adoptar compromisos y asumir responsabilidades compartidas. 


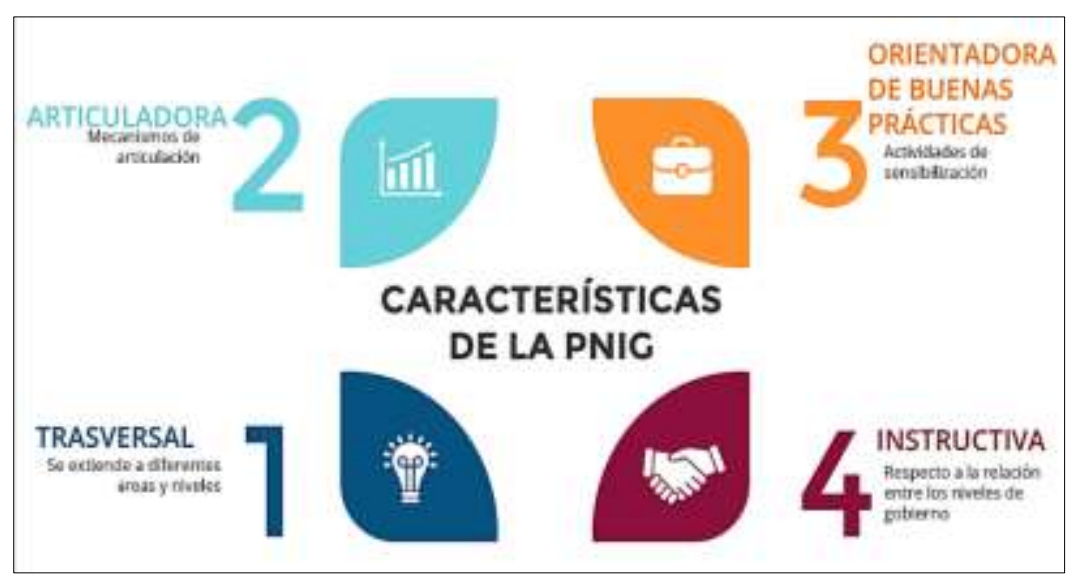

Figura 1. Características de la Política Nacional de Igualdad de Género.

Fuente: Elaboración propia en base a las entrevistas aplicadas.

Respecto al objetivo de analizar la implementación de la política nacional de igualdad de género, de acuerdo a los resultados obtenidos en la aplicación de la entrevista, se logró entender que, para implementar la política nacional de igualdad de género, se realizó un trabajo articulado, con relación a este punto, (Cotrina, 2020), establece que se ha venido trabajando de manera articulada con el gobierno regional, a través de las gerencias de producción, transportes y comunicaciones, educación, salud, cultura, parece mentira, pero se ha realizado gestión, (Nuñez, 2020), establece que desde el gobierno regional se ha implementado un plan de igualdad de género. Del mismo modo, como parte de la política se implementaron buenas prácticas en las instituciones educativas, con respecto a este punto, (Sánchez, 2020), establece que se implementaron acciones consecutivas sobre eventos en el ámbito de instituciones, donde se resalta el trabajo de los niños, niñas y adolescentes respecto a la promoción de la igualdad, que pronto se tornará en buenas prácticas, y según (Soriano, 2020), se ha implementado buenas prácticas en los concursos y proyectos que desarrollan las Instituciones Educativas en los tres niveles de educación. La aplicación de la política trajo consigo experiencias exitosas, dentro de las cuales se tuvo festivales regionales, provinciales y distritales justos sobre la presentación de la presencia de las buenas prácticas y al realismo estudiantil (Salazar, 2020), (Nuñez, 2020), mencionó que gracias al asesoramiento de una ONG del Centro Esperanza, en cuanto al enfoque de género e interculturalidad, se concretó una ordenanza regional sobre el fomento de la educación a las niñas y adolescentes rurales. Y finalmente, gracias a la implementación de esta política, se redujeron las brechas de género en el sector educación, así establece (Jiménez, 2020), cuando consideró que hay serios avances que 
ha costado mucho trabajo, pero todavía falta mucho por hacer, sobre todo en Ferreñafe que abarca Instituciones Educativas en Incahuasi, Cañaris, lugares donde el machismo y los estereotipos de género se encuentran arraigados.

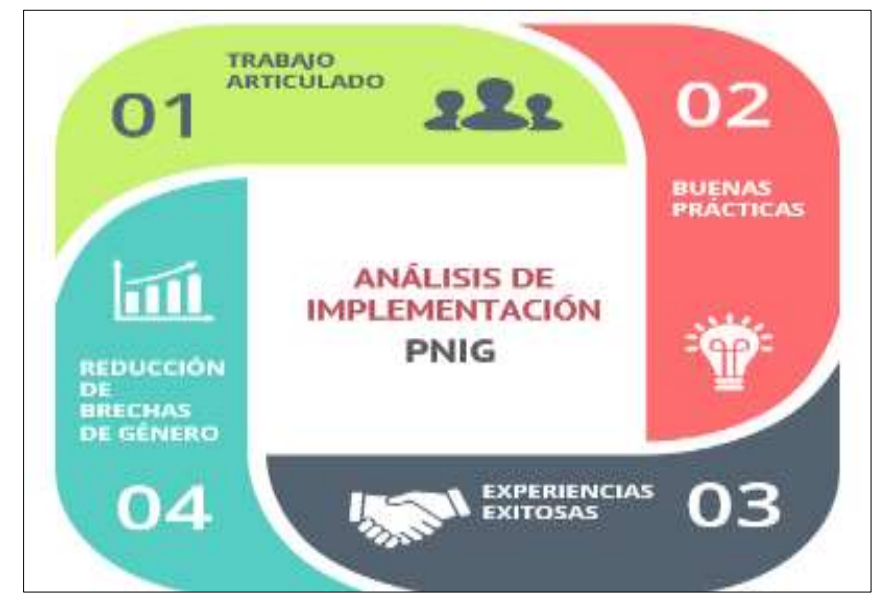

Figura 2. Análisis de aplicación de la Política Nacional de Igualdad de Género.

Fuente: Elaboración propia en base a las entrevistas aplicadas.

Con relación a seleccionar las teorías que influyen en la implementación de la política nacional de igualdad de género y el sistema educativo en la Región Lambayeque, de acuerdo a la revisión de la literatura existente, se obtuvo como resultado que, las teorías seleccionadas que estarían influyendo en la implementación de la PNIG, serían la Teoría Sociocultural, sustentada por Vygotsky, (1996), el Enfoque Feminista y el Enfoque de Derechos Humanos en las diversas políticas de género. Vygotsky, sobre la Teoría Sociocultural, afirma que el proceso de los seres humanos, está profundamente unido a su interrelación con el entorno social, histórico y cultural, siendo que a través de ello se cumplen funciones psicológicas de modo óptimo, idóneas del ser humano, como son la memoria, la observación voluntaria, el raciocinio y la resolución de taras, de igual manera, sostiene esta teoría que el desarrollo y desenvolvimiento ontogenético de la psiquis del varón está resuelto por las diversas fases de adquisición de las formas históricas y sociales de la cultura, citado por (Gutiérrez Rocha et al., 2017), de acuerdo a este concepto, sería inevitable una práctica pedagógica con una representación política y social organizada que conlleve el cambio que se requiere en la sociedad, en razón de ello, el maestro deberá adecuar su práctica educativa precisando la realidad, tomando en cuenta también la clase de alumno, sus aprendizajes previos, grado de madurez, para preparar su lección. En síntesis, esta teoría presenta la realidad sociocultural donde está insertado el alumno, el cual amolda su misma composición mental. Con relación al abordaje de la PNIG, en 
relación con la CNEBR, el Enfoque Feminista, fundamenta la relación del desarrollo de la educación e investigación con una visión feminista, por lo que, desde este enfoque, se pretende señalar que la investigación es perpetrar en una colección de procedimientos con orientación a otorgar contestación a las interrogantes superpuestas, con el objetivo de comprender o describir un hecho, sea cual sea la naturaleza de este. En ese sentido, recalca que, la investigación feminista, apareció ante la urgencia de hallar la condición de las mujeres, valga decir también su exclusión dentro del dominio científico, en ese sentido este enfoque lleva a conocimiento de la finalidad esencial de la investigación feminista que es producir discernimientos que logren dar cuenta, entre otras temáticas, del embrollo de las circunstancias de género que implican a mujeres y varones. Con relación al Enfoque de Derechos Humanos en las diversas políticas de género, (Meza Pavletich, 2016, p. 29) explica que los derechos los seres humanos tienen su origen en la exploración del valor de la dignidad y la libertad de la persona autónomamente de la correspondencia que las naciones pudieron haber realizado de los mismos. Por lo que, el análisis y la proyección de las políticas públicas sobre todo en igualdad de la mujer no se ve importante y resaltante, sino hasta que recién cuando el Estado Peruano incluye esta temática tan importante en su almanaque público; no cabe duda, es muy factible que la labor antepuesta en esas políticas fuesen los razonamientos indispensables para obtener menciana inclusión. Esto quiere decir que al respetar los derechos humanos en el ámbito supranacional requiere de un esfuerzo por trabajar en sus principios, más aún si una sociedad no los respeta íntegramente.

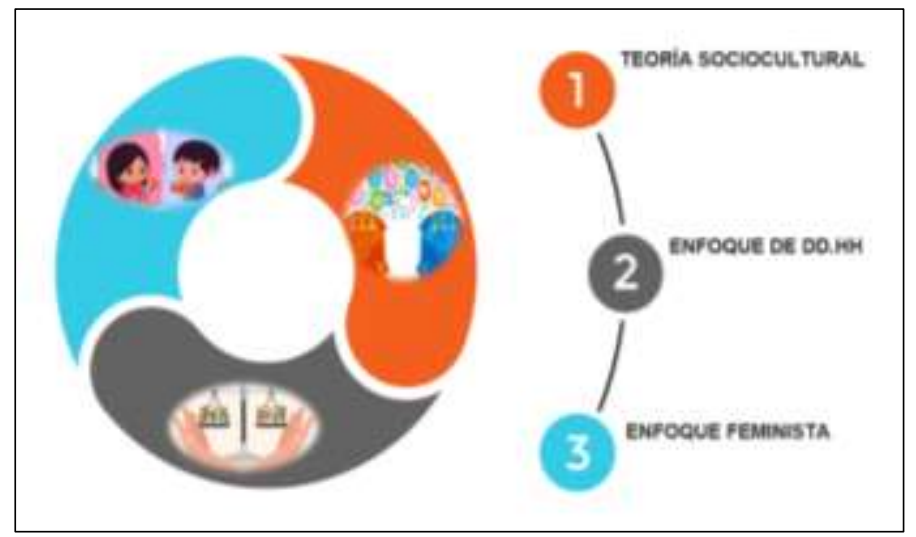

Figura 3. Teorías que influyen en la Política Nacional de Igualdad de Género.

Fuente: Elaboración propia en base a la revisión de la literatura existente. 
En relación con establecer cuál es la relación que existe entre las dimensiones de política nacional de igualdad de género y las dimensiones del currículo nacional de la educación básica regular en la Región Lambayeque, de acuerdo a los resultados obtenidos por medio de la aplicación del software SPSS 25, se obtuvo como coeficiente de correlación de Spearman para cada relación entre las dimensiones, el siguiente valor: entre la dimensión “educación con enfoque de género" y dimensión "enfoques transversales" $=0.694 *$, entre la dimensión "educación con enfoque de género" y dimensión “implementación del enfoque de igualdad de género en el currículo nacional de educación básica regular" = $0.808^{* *}$, entre la dimensión "implementación de la PNIG" y dimensión "enfoques transversales" $=0.492 * *$, entre la dimensión "implementación de la PNIG" y dimensión “implementación del enfoque de igualdad de género en el currículo nacional de educación básica regular" $=0.676^{* *}$, entre la dimensión "evaluación y seguimiento" y dimensión "enfoques transversales" $=0.658^{* *}, \mathrm{y}$, entre la dimensión "evaluación y seguimiento" y dimensión "implementación del enfoque de igualdad de género en el currículo nacional de educación básica regular" $=0.810^{* *}$. El nivel de significancia para todas estas correlaciones fue igual a 0.000 , es decir, la correlación fue significativa al 0.01, que es al $1 \%$, para todos los casos. Entonces, entre la dimensión "educación con enfoque de género" y dimensión "enfoques transversales" se infiere una correlación moderada; entre la dimensión "educación con enfoque de género" y dimensión "implementación del enfoque de igualdad de género en el currículo nacional de educación básica regular" se infiere una correlación alta; entre la dimensión "implementación de la PNIG” y dimensión "enfoques transversales" se infiere una correlación media; entre la dimensión “implementación de la PNIG" y dimensión "implementación del enfoque de igualdad de género en el currículo nacional de educación básica regular" se infiere una correlación moderada; entre la dimensión "evaluación y seguimiento" y dimensión "enfoques transversales" se infiere una correlación moderada; y, entre la dimensión "evaluación y seguimiento" y dimensión "implementación del enfoque de igualdad de género en el currículo nacional de educación básica regular" se infiere una correlación alta. Todas las dimensiones presentan nivel de correlación, y de tipo positiva.

Tabla 1. Correlación entre las Dimensiones de Educación con enfoque de género, Implementación y Evaluación y seguimiento de la Variable 1 y las Dimensiones de 
Enfoques transversales e Implementación del enfoque de igualdad de género de la Variable 2

\begin{tabular}{|c|c|c|c|c|c|c|c|}
\hline & & & D1V1 & D2V1 & D3V1 & D1V2 & D2V2 \\
\hline \multirow{15}{*}{$\begin{array}{l}\text { Rho de } \\
\text { spearman }\end{array}$} & \multirow{3}{*}{ D1V1 } & Coeficiente de correlación & 1,000 &, $582^{* *}$ &, $792^{* *}$ & $694^{* *}$ &, $808^{* *}$ \\
\hline & & Sig. (bilateral) & - & ,000 &, 000 &, 000 & 000 \\
\hline & & $\mathrm{N}$ & 45 & 45 & 45 & 45 & 45 \\
\hline & \multirow{3}{*}{$\mathrm{D} 2 \mathrm{~V} 1$} & Coeficiente de correlación &, $582^{* *}$ & 1,000 &, $701^{* *}$ &, $492^{* * *}$ &, $676^{* *}$ \\
\hline & & Sig. (bilateral) &, 000 & . & ,000 &, 001 & 000 \\
\hline & & $\mathrm{N}$ & 45 & 45 & 45 & 45 & 45 \\
\hline & \multirow{3}{*}{ D3V1 } & Coeficiente de correlación &, $792^{* *}$ &, $701^{* *}$ & 1,000 &, $658^{* *}$ &, $810^{* *}$ \\
\hline & & Sig. (bilateral) &, 000 &, 000 & - &, 000 & 000 \\
\hline & & $\mathrm{N}$ & 45 & 45 & 45 & 45 & 45 \\
\hline & \multirow{3}{*}{ D1V2 } & Coeficiente de correlación &, $694^{* *}$ &, $492^{* *}$ &, $658^{* *}$ & 1,000 &, $729^{* *}$ \\
\hline & & Sig. (bilateral) &, 000 & 001 &, 000 & . & 000 \\
\hline & & $\mathrm{N}$ & 45 & 45 & 45 & 45 & 45 \\
\hline & \multirow{3}{*}{$\mathrm{D} 2 \mathrm{~V} 2$} & Coeficiente de correlación &, $808^{* *}$ &, $676^{* *}$ &, $810^{* *}$ &, $729^{* *}$ & 1,000 \\
\hline & & Sig. (bilateral) &, 000 &, 000 & ,000 &, 000 & \\
\hline & & $\mathrm{N}$ & 45 & 45 & 45 & 45 & 45 \\
\hline
\end{tabular}

Fuente: Elaboración propia en el software SPSS 25 con base en las encuestas aplicadas.

Con relación a la determinación del nivel de influencia de la política nacional de igualdad de género en el currículo de la educación básica regular en la Región Lambayeque, de acuerdo a los resultados obtenidos por medio de la aplicación del software SPSS 25, se obtuvo como coeficiente de correlación de Spearman el valor de $0.845^{* *}$, entre la variable 1 que obedece a la política nacional de igualdad de género y la variable 2 que obedece al currículo de la educación básica regular en la Región Lambayeque, con un nivel de significancia igual a 0.000 , es decir, la correlación fue significativa al 0.01, que las variables de estudio, esto es, al 1\%. De este resultado se infiere que, existe correlación alta, y positiva, entre las variables de estudio.

Tabla 2. Correlación entre la Variable 1 Política Nacional de Igualdad de Género PNIG y la Variable 2 Currícula Nacional de Igualdad de Género - CNEBR 


\begin{tabular}{|c|c|c|c|c|}
\hline & & & PNIG & CNIG \\
\hline \multirow{6}{*}{$\begin{array}{l}\text { Rho de } \\
\text { Spearman }\end{array}$} & \multirow{3}{*}{ PNIG } & Coeficiente de correlación & 1,000 &, $845^{* *}$ \\
\hline & & Sig. (bilateral) & . & ,000 \\
\hline & & $\mathrm{N}$ & 45 & 45 \\
\hline & \multirow{3}{*}{$\mathrm{CNIG}$} & Coeficiente de correlación &, $845^{* *}$ & 1,000 \\
\hline & & Sig. (bilateral) & ,000 & . \\
\hline & & $\mathrm{N}$ & 45 & 45 \\
\hline
\end{tabular}

Fuente: Elaboración propia en el software SPSS 25 en base a las encuestas aplicadas.

El resultado final del análisis del cumplimiento de cada objetivo, conlleva validar la hipótesis que establece que la política pública de igualdad de género tiene influencia positiva en el currículo nacional de la educación básica regular en la Región Lambayeque.

\section{DISCUSIÓN}

De acuerdo a los datos obtenidos de los instrumentos aplicados y el análisis respectivo, se llegó a obtener como cumplimiento de los objetivos de la manera en que se explicará y discutirá a continuación:

Con relación al objetivo específico 1, en donde se trataba de distinguir las características de la política pública de igualdad de género en la Región Lambayeque, se apreció que las características identificadas fueron las siguientes: La política pública de igualdad de género es transversal, articuladora, orientadora de buenas prácticas, e instructiva. La investigación de (Espino y Salvador, 2016, p. 14), habla sobre la característica transversal de la PNIG, y coincide con el análisis de resultados, puesto que establece que, no hay forma de ver el enfoque de género de manera aislada, sino de forma transversal, en cada una de sus fases, en la implementación de las políticas, programas, planes y estrategias. El (Decreto Supremo N 008 -2019-MIMP - Política Nacional de Igualdad de Género, s. f., p. 13), al hablar de enfoque de género dentro de las políticas públicas, establece que este instrumento determina las desigualdades y falta de equidad en las relaciones sociales entre géneros, partiendo de determinar cuáles serían las obligaciones (tarea o rol asignados) para mujeres y para hombres dentro de la sociedad donde se encuentran. Luego de identificar las causas que originan los tratos desiguales entre géneros, en las 
diversas culturas y sociedad, se puede dar uso al enfoque de género, puesto que este enfoque sirve para acabar con las brechas generadas a través de los tratos desiguales entre hombres y mujeres, con la implementación de medidas que contribuyan en la lucha en contra de la falta de equidad y violencia de género, y contra todo tipo de rasgo o característica humana que esté generando algún trato desigual, otorgándole a ambos géneros la igualdad necesaria para obtener el acceso indistinto a cada servicio y recurso que le es otorgado a las personas a nivel nacional, con esta descripción de del Decreto Supremo citado, podemos apreciar las características del procedimiento seguido para dar nacimiento a una política pública que tenga enfoque de género, lo que sería necesario a tomar en cuenta en comparativo con las características del PNIG.

En relación con el objetivo específico 2, que se trataba del análisis de la implementación de la política nacional de igualdad de género, se observó que para implementar la política nacional de igualdad de género, se hizo un trabajo articulado con los niveles del estado, y todas las instituciones involucradas, asimismo, se implementaron buenas prácticas en las instituciones educativas con la participación activa de los actores principales, se pudieron percibir que la política trajo consigo experiencias exitosas, dentro de las cuales se tuvo festivales regionales, provinciales y distritales sobre la presentación de la presencia de las buenas prácticas y al realismo estudiantil, entonces, se puede agregar que, gracias a la implementación de la PNIG, se encuentra reduciendo las brechas de género en el sector educación. Con relación a los resultados obtenidos en este objetivo, se encuentra aval en la Estrategia de Montevideo para la Implementación de la Agenda Regional de Género en el Marco del Desarrollo Sostenible hacia 2030, 2016 (Biblioteca de la CEPAL - Comisión Económica para América Latina y el Caribe, s. f.), puesto que en esta investigación se determina que es importante que la ejecución de las políticas públicas sea de manera colateral, es decir, con un trabajo articulado, hacia los tres niveles de gobierno y a todas las instituciones tanto públicas como privadas, siendo que de nada sirve una estrategia o táctica cuando no se planifica el impacto en los agentes del cambio, que finalmente harán que ésta funcione o quede en teoría y papel. Asimismo, (Espino y Salvador, 2016, p. 14), establecen que la implementación de la PNIG se implementa con la finalidad de conseguir mejores resultados en el cierre de brechas de desigualdad, por ende, suba el nivel de dignidad. De forma opuesta el Portal Web de la Organización de las Naciones Unidas, (s. f.), señala que entre los años 2000 y 2015 existió acercamiento 
a la igualdad entre los géneros, sin embargo, aún las mujeres y las niñas siguen sufriendo la injusticia y la violencia en todos los sectores de la tierra, es decir, para la ONU, las brechas aún no se habrían terminado, a pesar del desarrollo de políticas en pro de ello.

Respecto a la relación del objetivo específico 3, sobre la selección de las teorías que influyen en la implementación de la política nacional de igualdad de género y el sistema educativo en la Región Lambayeque, luego de realizar la revisión de la literatura existente, se pudo determinar cómo las teorías que estarían influyendo en la implementación de la PNIG y el sistema educativo son la Teoría Sociocultural, sustentada por Vygotsky, (1996), el Enfoque de Derechos Humanos en las diversas políticas de género y el Enfoque Feminista. Al respecto el (Ministerio de la Mujer y Poblaciones Vulnerables, s.f.) establece que es imprescindible el planeamiento y ejecución con impacto de una política pública que desde un apunte multisectorial y de género, proyecte básicamente las principales formas de la discriminación y la diferenciación que perturba de manera principal a las mujeres, quienes constituyen el 50,8\%, de la población en el país, es decir, más de la mitad, sobreviven con límites al goce de sus derechos fundamentales y sobre todo, oportunidades de prosperidad y desarrollo, circunstancias que requieren de una contundente diligencia e intervención desde el Estado para impedir su persistente regeneración social y transferencia al interior de las generaciones, en estas líneas el MIMP, establece los criterios a tomar en cuenta para establecer una política que luche contra las brechas discriminatorias, y habla de los derechos fundamentales, es decir, toma en consideración un Enfoque de Derechos Humanos que tendría que ser aplicada en la política de género, que luchara contra el trato diferente y discriminatorio, que es precisamente una de los enfoques que influye en la PNIG.

En cuanto al objetivo específico 4, que buscaba establecer cuál es la relación que existe entre las dimensiones de política nacional de igualdad de género y las dimensiones del currículo nacional de la educación básica regular en la Región Lambayeque, se pudo establecer por medio de la tabla 1 que, entre la dimensión "educación con enfoque de género" y dimensión "enfoques trasversales” existía una correlación moderada; entre la dimensión "educación con enfoque de género" y dimensión "implementación del enfoque de igualdad de género en el currículo nacional de educación básica regular" existía una correlación alta; entre la dimensión “implementación de la PNIG” y dimensión “enfoques trasversales" existía una correlación media; entre la dimensión "implementación de la 
PNIG” y dimensión “implementación del enfoque de igualdad de género en el currículo nacional de educación básica regular" existía una correlación moderada; entre la dimensión "evaluación y seguimiento" y dimensión "enfoques trasversales" existía una correlación moderada; $y$, entre la dimensión "evaluación y seguimiento" y dimensión “implementación del enfoque de igualdad de género en el currículo nacional de educación básica regular" existía una correlación alta. Por ese resultado, todas las dimensiones eran correlacionales, en diferentes medidas, y de tipo positiva. Con respecto a la correlación de las variables podemos mencionar a (Eras Cabrera y Ycaza Maridueña, 2019), que establecen que, la relación que existe entre el enfoque de género y la educación, definitivamente es la articulación entre los directivos y docentes, quienes aportan de manera directa e imparcial, en la relación que debe existir al interior de las aulas, de tal manera que se pongan en evidencia las relaciones existentes, con la finalidad de contribuir hacia los cambios paradigmas, superar las desigualdades y posibles conflictos que se originan mediante la convivencia escolar. En ese contexto, el autor resalta que los maestros presentan inconformidad ante la resolución de situaciones relacionadas con el enfoque de género, siendo que manifiestan la insuficiente capacitación sobre estos temas, la cual es suma importancia, necesaria y oportuna en estos casos, por ejemplo fue común observar y comprobar que surgen inconvenientes para los docentes ante situaciones diarias y cotidianas entre los jóvenes que no trabajan con aquellas adolescentes que son madres a temprana edad, ya que mencionan que ellas no realizan con responsabilidad las tareas encomendadas en clase. p. 76. También es importante mencionar al (Decreto Supremo Nº 008 -2019-MIMP - Política Nacional de Igualdad de Género, s. f.), y su descripción de la orientación de la PNIG, nos especifica que esta política busca disminuir progresivamente las brechas de género existentes, tales como el empleo, ingresos, uso del tiempo, participación, entre otras, así como abordar las principales causas de la discriminación y la desigualdad que afecta principalmente a las mujeres, quienes constituyendo el 50,8\% de la población, viven con un limitado ejercicio de sus derechos fundamentales y oportunidades de desarrollo, situación que requiere de una decidida intervención desde el Estado para impedir su sistemática reproducción social y transmisión intergeneracional. Y siendo esta la orientación de la PNIG, establece que, es indispensable saber la forma de seguimiento y evaluación de la PNIG, en ese sentido, el (Decreto Supremo Nº 008 -2019-MIMP - Política Nacional de Igualdad de Género, s. f.), 
establece que el seguimiento se realiza a través del aplicativo informático CEPLAN V.01, el cual permite generar reportes de seguimiento estandarizados. Como herramienta adicional, los indicadores de la PNIG se incorporan en el tablero de desempeño del MIMP, el cual está diseñado para hacer seguimiento al desempeño de las políticas, planes especiales multisectoriales, programas y proyectos, en el cumplimiento de las metas establecidas referidas a sus indicadores estratégicos, gestión financiera y administrativa, y otros. De igual manera, la evaluación de esta implementación, tiene periodicidad semestral y anual, y contiene la información provista por los ministerios encargados de brindar los servicios previstos en la PNIG y por el Instituto Nacional de Estadística e Informática (INEI), datos que arrojan definitivamente indicadores de reducción de las brechas existentes en educación. Como se puede apreciar, las dimensiones del enfoque de género deben son determinantes, y su cumplimiento en su implementación es básica, entonces deben ir a la par con las dimensiones del currículo nacional de educación básica regular, con el fin de lograr el objetivo que pretende la PNIG en el área educativa a nivel nacional.

Con relación al objetivo general, la determinación del nivel de influencia de la política nacional de igualdad de género en el currículo de la educación básica regular en la Región Lambayeque, de acuerdo a los resultados obtenidos se establece que existe correlación alta, y positiva, entre las variables de estudio, debido al coeficiente de correlación de Spearman el valor de $0.845^{* *}$, con un nivel de significancia igual a 0.000 , de acuerdo a la tabla 2. El (MIMP, 2019) establece que la PNIG, es la incorporación del enfoque de género en las políticas públicas como una herramienta de análisis es fundamental porque permita identificar los roles y tareas que realizan los hombres y las mujeres en una sociedad, así como las asimetrías, relaciones de poder e inequidades que se producen entre ellos. Del mismo modo, y, el Currículo Nacional de Educación Básica, se define como el documento que establece el Perfil de Egreso de la Educación Básica, las competencias nacionales y sus progresiones desde el inicio hasta el fin de la educación básica, así como sus niveles esperados por ciclo, nivel y modalidades. (Currículo Nacional de la Educación Básica, 2016). De acuerdo a la actualidad social, se estaría incorporando este enfoque de género, al Currículo Nacional de educación Básica, y, así lo establece el mismo documento, puesto que menciona que, a nivel nacional se viene aplicando el Enfoque igualdad de género, para lograr que las diversas conductas y 
necesidades en todos los casos, tengan una misma valoración sin distinciones por género. Con este enfoque se busca que se acabe la desigualdad, para que los roles, deberes y derechos, y también las circunstancias que generen alguna oportunidad aprovechable, no obedezcan a géneros, y todos puedan acceder con igualdad y alcancen el desarrollo a nivel social, obteniendo los beneficios sin distinciones. Replantear el uso de los términos "femenino" o "masculino", usándolos solo para marcar una diferencia biológica, más no deben ser de uso para generar distinciones o tratos desiguales. Se debe dejar de seguir los antiguos paradigmas con lo que se generan desigualdades, como por ejemplo pensar que una mujer debe quedarse en casa para encargarse de la casa, dejando de lado su formación educativa. (Currículo Nacional de la Educación Básica, 2016, p. 19). Finalmente, se cita la tesis planteada por (Rodríguez Reyes, 2018), que refiere, que a todas luces, existe la transmisión de los dogmas de los maestros centrados en aplicación a la sexualidad, el sexo y el género, en el aula hacia sus alumnos, para plantear el adiestramiento y enseñanza sexual; asimismo, (Zemzylina, 2017, p. 43), en su investigación, establece que la implementación de la educación basada en género dará como producto la formación de la autoconciencia de género entre los adolescentes y jóvenes que se materializa, a través de la comprensión de la igualdad de ambos sexos en lo social y vida política; posición activa con respecto a la implementación de cambios transformacionales de género en la República de Ucrania; así como capacidad de compromiso, tolerancia en diferentes situaciones de la vida real es decir, ambas investigaciones, relaciona la enseñanza en aulas con el enfoque de género, mostrando que si existiría una influencia de tipo positiva, en la PNIG en el Currículo Nacional de Educación Básica, avalando el resultado obtenido.

La metodología escogida cabía dentro del enfoque cualitativo socio crítico fenomenológico, puesto que buscaba captar la percepción de las personas, sobre el sentido o explicación de determinado fenómeno y evaluar su experiencia en el mismo (Hernández Sampieri et al., s. f., p. 515), de acuerdo a esta definición se tuvo que recoger las apreciaciones de personas que se enfrentan a la realidad que existe sobre el fenómeno o problema estudiado, llevar a la práctica este enfoque se volvió un hecho limitado luego de lo que ocurrió a nivel mundial, tras salir a la luz la enfermedad COVID19, que, debido a su gran impacto, se volvió pandemia. Esta realidad social por la que todo el mundo atravesó, origino que no se pueda recoger las apreciaciones de los expertos o las personas directamente relacionadas con el tema de investigación, de forma presencial, se tuvo que 
hacer uso de las redes sociales, y la tecnología, del mismo modo, se realizó ajustes a la forma en que se venía desarrollando la tesis. De acuerdo al enfoque mixto, se tuvo que aplicar los instrumentos, tanto encuestas, como la entrevista, esta aplicación se realizó tomando en cuenta las circunstancias sociales actuales. Siendo así, las técnicas utilizadas para la recolección de datos, fueron validadas por especialistas en el área que tenía relación con las variables de estudio, con la finalidad de lograr resultados confiables que contribuyan de manera significativa a la investigación.

Esta investigación, luego de su desarrollo completo, muestra que existe necesidad de incluir el enfoque de género como parte de la PNIG, en el Currículo Nacional de Educación Básica, tomando en cuenta el desarrollo social del ser humano en el mundo, y la necesidad de acabar con los actos discriminatorios basados en el género de la persona. De esta forma la sociedad logrará un avance igualitario para todos sus integrantes, en todos los aspectos, partiendo por el aspecto educacional.

\section{CONCLUSIONES}

Se logró distinguir a partir del análisis de cada entrevista a expertos que, la PNIG en la Región Lambayeque, tiene cuatro características identificadas desde su implementación: transversal, articuladora, orientadora de buenas prácticas e instructiva, contrastado con antecedentes notables y revisión bibliográfica.

Se determinó que para la implementación de la política nacional de igualdad de género en la Región Lambayeque, se hizo un trabajo articulado con los tres niveles de gobierno, y el compromiso de todas las instituciones, para lo cual, se pudo percibir que esta política trajo consigo experiencias exitosas y la presencia de buenas prácticas respecto al enfoque de género, por lo que gracias a la implementación de la PNIG, la reducción de brechas de género en el sector educación ha sido considerable, sin embargo, tal como lo manifiestan dos expertos aún falta mucho para desterrar la desigualdad de género.

Se concluyó que las teorías y enfoques influyentes en la implementación de la PNIG y el sistema educativo son la Teoría Sociocultural, el Enfoque de Derechos Humanos y el Enfoque Feminista, sin embargo, la investigación en respaldo por lo manifestado por el MIMP, establece los criterios a tomar en cuenta para establecer una política que luche contra las brechas discriminatorias, y apunta a los derechos fundamentales, es decir, considera un Enfoque de Derechos Humanos. 
Se estableció que las dimensiones del enfoque de género deben son determinantes, y cumplimiento en su implementación es básica, entonces deben ir a la par con las dimensiones del currículo nacional de educación básica regular, con el fin de lograr el objetivo que pretende la PNIG en el área educativa a nivel nacional, sin embargo cabe recalcar la posición de la directora de Gestión Pedagógica de la UGEL Ferreñafe, quien manifestó no incorporar en su planificación curricular al enfoque de género debido a los serios enfrentamientos por su incorporación.

Se determinó de acuerdo a los resultados obtenidos que existe correlación alta, y positiva, entre las variables de estudio, debido al coeficiente de correlación de Spearman el valor de $0.845^{* *}$, con un nivel de significancia igual a 0.000 , de acuerdo a la tabla 2 .

\section{LISTA DE REFERENCIAS}

Ashworth, F., Brennan, G., Egan, K., Hamilton, R., y Sáenz, O. (2004). Learning Theories and Higher Education. Level 3, 2(1), 1-17. https://doi.org/10.21427/D7S43V

Biblioteca de la CEPAL - Comisión Económica para América Latina y el Caribe, C. (s. f.). Biblioguias: Agenda Regional de Género: Agenda Regional de Género. Recuperado 30 de mayo de 2020, de https://biblioguias.cepal.org/AgendaRegionalGenero/Buscador

Cotrina, M. (2020). Directora de Gestión Pedagógica. (E. Segura, Entrevistador)

Cuero, C. (2020). The COVID-19 pandemic. 2. http://access.revistasmedicas.org/pdf/?opensource=rev\&openpdf=y\&ojl=aHR0c DovL29qcy5yZXZpc3Rhc211ZGljYXMub3JnL2luZGV4LnBocC9ybWRwL2F ydGljbGUvdmlld0ZpbGUvODcyLzgxOA==\&o=MDAtZWRpdG9yaWFsLnBk

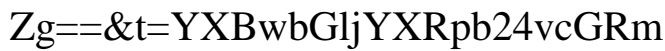

Currículo Nacional de la Educación Básica. (2016). Currículo Nacional| Minedu. http://www.minedu.gob.pe/curriculo/

Decreto Supremo N 008 -2019-MIMP - Política Nacional de Igualdad de Género. (s. f.). Recuperado 31 de mayo de 2020, de https://cdn.www.gob.pe/uploads/document/file/305292/ds_008_2019_mimp.pdf

Eras Cabrera, M., y Ycaza Maridueña, J. E. (2019). Enfoque de género en el proceso educativo: Propuesta: Campaña Social [Universidad de Guayaquil. Facultad de Filosofía, Letras y Ciencias de la Educación]. http://repositorio.ug.edu.ec/handle/redug/41623 
Espino, A., y Salvador, S. (2016). Semáforo de género de las políticas públicas que promueven el empoderamiento económico de las mujeres. https://idl-bncidrc.dspacedirect.org/handle/10625/55880

Fuster Guillen, D. E. (2019). Investigación cualitativa: Método fenomenológico hermenéutico. Propósitos y Representaciones, 7(1), 201-229. https://doi.org/10.20511/pyr2019.v7n1.267

Gutiérrez Rocha, R. D. C., Mármol Silva, A., y Mozo Florez, K. M. (2017). Impacto de la pedagogía de género como propuesta pedagógica para el desarrollo de las $\begin{array}{llll}\text { competencias lectora } & y & \end{array}$ http://manglar.uninorte.edu.co/bitstream/handle/10584/7666/130238.pdf?sequen $\mathrm{ce}=1$

Hernández Sampieri, R., Fernández Collado, C., y Baptista Lucio, M. del P. (s. f.). Metodología de la investigación (Quinta Edición). McGRAW-HILL / INTERAMERICANA EDITORES, S.A. DE S.V. https://www.esup.edu.pe/descargas/dep_investigacion/Metodologia\%20de\%20la \%20investigaci\%C3\%B3n\%205ta\%20Edici\%C3\%B3n.pdf

Huerta Rojas, F. (2017). Foro Consultivo Internacional: Enseñanza de las Ciencias en Preescolar con Enfoque de Género-UNESCO Biblioteca Digital. UNESDOC Biblioteca Digital. https://unesdoc.unesco.org/ark:/48223/pf0000260750?posInSet=1\&queryId=NEXPLORE-92ab083a-2134-4959-94e5-d6ba59e227e4

Jiménez, G. (2020). directora de UGEL Ferreñafe. (E. Segura, Entrevistador)

Nuñez, J. (2020). Gerenta Regional de Programas Sociales del Gobierno Regional de Lambayeque. (E. Segura, Entrevistador)

Meza Pavletich, E. I. (2016). Análisis del Plan Nacional de igualdad de género con énfasis en la dimensión de orientación sexual: Aportes de la gerencia social para mejorar las políticas de género en el Perú [Pontificia Universidad Católica del Perú]. http://tesis.pucp.edu.pe/repositorio/handle/20.500.12404/6633

Ministerio de la Mujer y Poblaciones Vulnerables. (s. f.). Decreto Supremo N ${ }^{\circ}$ 008-2019MIMP. Recuperado 31 de mayo de 2020, de https://www.gob.pe/institucion/mimp/normas-legales/271118-008-2019-mimp 
Portal Web de la Organización de las Naciones Unidas, O. de D. S. (s. f.). Igualdad de género y empoderamiento de la mujer. Desarrollo Sostenible. Recuperado $30 \mathrm{de}$ mayo de 2020, de https://www.un.org/sustainabledevelopment/es/genderequality/

Portal Web del Gobierno Regional de Lambayeque, U. D. G. E. L. L. (s. f.). UNIDAD DE GESTIÓN EDUCATIVA LOCAL LAMBAYEQUE. Recuperado 30 de mayo de 2020, de https://www.regionlambayeque.gob.pe/web/instituciones?pass=MTY=

Rodríguez Reyes, A. L. (2018). Creencias docentes: El enfoque de género en la educación y la educación sexual en secundaria [Pontificia Universidad Católica del Perú]. http://tesis.pucp.edu.pe/repositorio/handle/20.500.12404/13039

Salazar, Á. (2020). Director de UGEL Chiclayo. (E. Segura, Entrevistador)

Soriano, E. (2020). Directora de UGEL Lambayeque. (E. Segura, Entrevistador)

Zemzylina, N. I. (2017). Gender approach in education: The search of methots and ways of implementation in Ukraine. Вісник Черкаського університету. Серія Історичні науки, № 1, 40-46. http://eprints.cdu.edu.ua/2931/ 